\title{
Reduction of the toxicity of tobacco smoke with catalytic materials: Study of the influence of SBA-15 synthesis variables, time and temperatura of the hydrothermal treatment.
}

\author{
Created by: Nerea Juárez-serrano, MAntonio Marcilla \\ Version received: 15 April 2020
}

check for updates

\begin{abstract}
This article is associated with the MDPI "Catalysts" journal, which belongs to a Special Issue "SBA-15 and Catalysis", "The Effect of Temperature and Time of the Hydrothermal Treatment in the SBA-15 Synthesis Process on the Structure and Textural Properties and the Ability to Reduce the Evolution of Tars in Tobacco Smoking" and the authors Juárez-Serrano, N. a; Asensio, J.a ; Martínez-Castellanos, I. ${ }^{\text {a }}$; Beltrán, M.I. $;$; and Marcilla A.*a
\end{abstract}

${ }^{a}$ Chemical Engineering Department. University of Alicante. Apartado 99, 03690, Alicante, Spain.

*Corresponding author: antonio.marcilla@ua.es

SBA-15 synthetized at different values of time and temperature of the hydrothermal treatment were mixture with tobacco in order to determine the capacity of reduction of toxic and carcirogenic compounds on tobacco smoke. The effect of temperature is not significative but time presents a remarked effect. And a parameter not frequently studied, the aparent density, has been shown the most relevant relation with the results on smoking experimentos. Finally, the effect of reduce the supernatant liquor also has been studied and the results have shown that the material properties remain practically unchanged.

\section{Introduction}

The main objective of the present work is to modify the time and temperature of the hydrothermal treatment during the synthesis of the SBA-15 for studying the influence of the corresponding properties developed on the emission of toxic compounds when smoking their mixtures with 3R4F reference tobacco. Nevertheless, we have not found commercial availability of SBA-15 synthesized modifying these variables and with different textural properties. Consequently, if such a study is to be developed, the corresponding samples must be "ad hoc" synthetised and characterized to relate the properties with the effect on tobacco smoke. On another hand, the hydrothermal treatment is usually carried out in autoclaves or tapered vessels. This fact may involve high cost of the equipment required for industrial applications, since large volume reactors capable of standing the developed pressures would be used. Thus, we have also considered the effect of removing the supernatant liquid before the hydrothermal treatment in order to explore the possibility of reducing the volume of the reactors, both objectives contributing to optimize and simplify the SBA-15 synthesis conditions.

\section{Definition}

During the last decades, great efforts have been made to reduce the consumption of tobacco, however, due to the great difficulty of completely eradicating tobacco consumption, different approaches to reduce the number of toxic compounds in tobacco smoke[1] have attracted great attention. In this sense, the use SBA-15 has been studied as a tobacco additive yielding high reductions of toxic compounds. Generally, its synthesis process involves 4 principal steps, i.e.: pre-treatment, 
hydrothermal treatment, drying and calcination. Lots of papers can be found on the effect of the synthesis conditions on the properties of the material, mainly during the first and second steps (i.e.: $[\underline{2}][\underline{3}][\underline{4}])$. The textural properties of the catalysts are likely to affect its behaviour un every particular application. Nevertheless, no studies from other research groups have been found on the influence of the different properties developed by the material on its effect in modifying the chemistry and reducing the presence of toxicant compounds in tobacco smoke.

\section{Data, Modelm Applications and Influences \\ - Characterization of SBA-15}

SBA-15 materials were prepared according to the synthesis described by Zhao et al. [ㅁ], varying the time and temperature of the hydrothermal treatment. Two variables were studied, temperature (TH) and time $(\mathrm{tH})$ of the hydrothermal process. The nomenclature of the samples is described as: $X / Y^{*}$ where $X$, is the time in hours; $\mathrm{Y}$, the temperature in ${ }^{\circ} \mathrm{C}$; and the symbol "*", represents the samples prepared without reducing the volume of the supernatant liquor.

Materials obtained have been characterized by $77 \mathrm{~K} \mathrm{~N} \mathrm{~N}_{2}$ isotherm, XRD, SEM and apparent density. Figures 1, 2 and 3 and Table 1 show the results obtained. The reduction of the volume of supernatant liquor has no significant effect on the properties developed. The shift of the XRD reflections to lower angles at high temperatures was also observed by other authors (i.e: []][]][ㅇ] $)$. Increasing the time of the hydrothermal treatment results in effects similar to increasing the temperature. The total pore volume and the pore diameter increase with increasing temperature. On the contrary, the BET surface area and

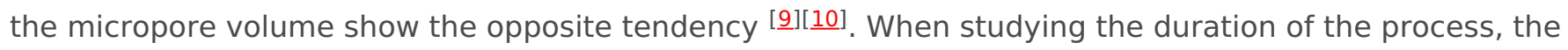
micro and mesoporosity are markedly increased as a consequence of the consolidation of the structure

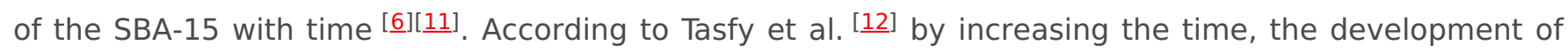
the self-assembly process could originate an increase in the total pore volume and a decrease in the volume of micropores. The temperature and the amount of supernatant liquor show almost significant influence on apparent density. Conversely, the time of hydrothermal treatment, causes a marked decrease in density, especially at shorter times. A clear qualitative correlation has been found between the morphology of the material and the apparent density, both also correlated with the effectivity in reducing the toxicants in tobacco smoke.

It can be concluded that the synthesized materials have developed typical properties of SBA-15 materials and the evolution of these characteristics is in accordance with the properties described by numerous authors. 

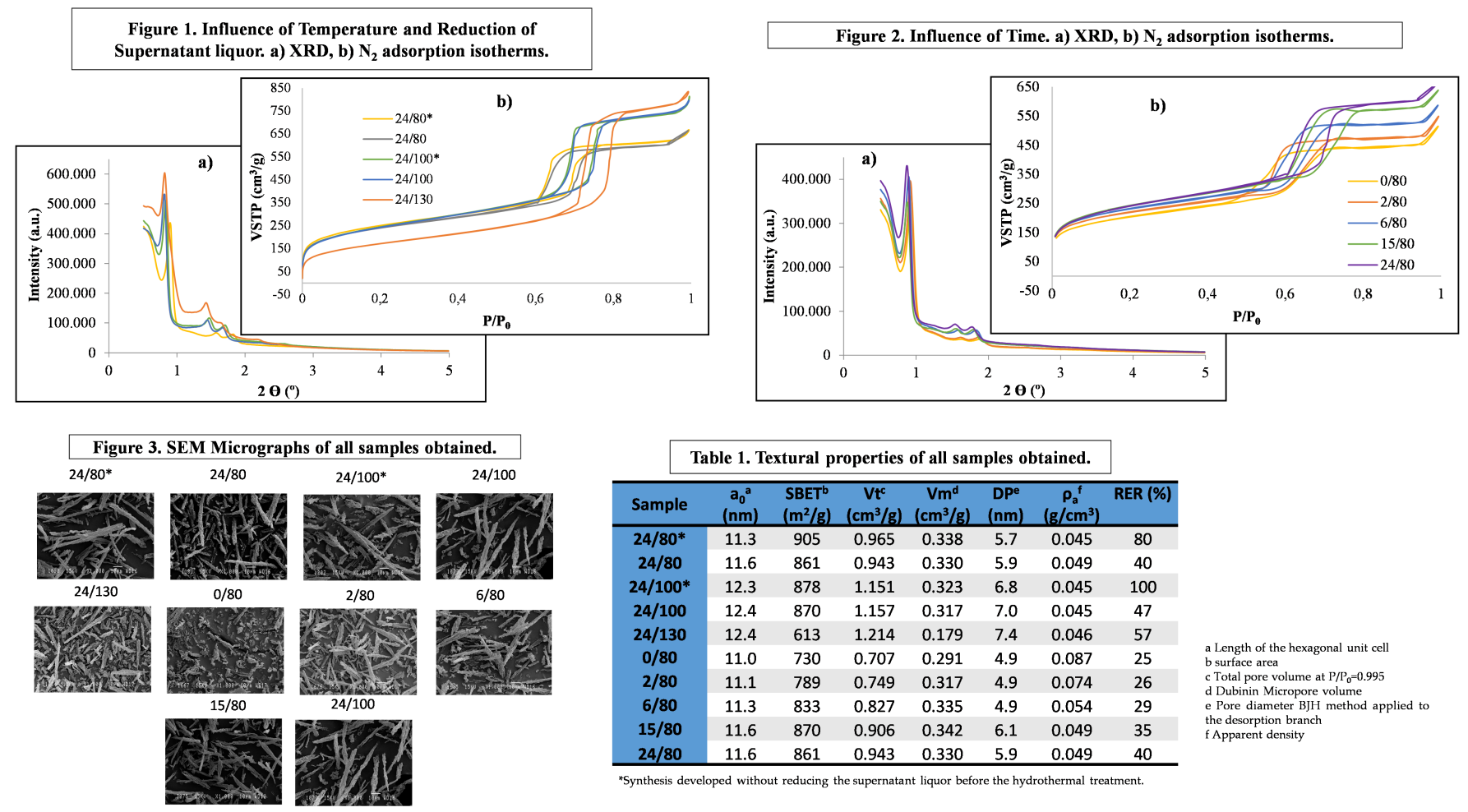

\begin{tabular}{|c|c|c|c|c|c|c|c|}
\hline Sample & $\begin{array}{c}a_{0}{ }^{a} \\
(n m))\end{array}$ & $\begin{array}{l}\text { SBET } \\
\left(\mathrm{m}^{2} / \mathrm{g}\right)\end{array}$ & $\begin{array}{c}\mathrm{Vt}^{\mathrm{c}} \\
\left(\mathrm{cm}^{3} / \mathrm{g}\right)\end{array}$ & $\begin{array}{c}\mathrm{Vm}^{\mathrm{d}} \\
\left(\mathrm{cm}^{3} / \mathrm{g}\right)\end{array}$ & $\begin{array}{c}\mathrm{DP}^{2} \\
(\mathrm{~nm})\end{array}$ & $\begin{array}{c}\rho_{\mathrm{a}}^{\prime} \\
\left(\mathrm{g} / \mathrm{cm}^{3}\right)\end{array}$ & RER (\%) \\
\hline $24 / 80^{*}$ & 11.3 & 905 & 0.965 & 0.338 & 5.7 & 0.045 & 80 \\
\hline $24 / 80$ & 11.6 & 861 & 0.943 & 0.330 & 5.9 & 0.049 & 40 \\
\hline $24 / 100^{*}$ & 12.3 & 878 & 1.151 & 0.323 & 6.8 & 0.045 & 100 \\
\hline $24 / 100$ & 12.4 & 870 & 1.157 & 0.317 & 7.0 & 0.045 & 47 \\
\hline $24 / 130$ & 12.4 & 613 & 1.214 & 0.179 & 7.4 & 0.046 & 57 \\
\hline $0 / 80$ & 11.0 & 730 & 0.707 & 0.291 & 4.9 & 0.087 & 25 \\
\hline $2 / 80$ & 11.1 & 789 & 0.749 & 0.317 & 4.9 & 0.074 & 26 \\
\hline $6 / 80$ & 11.3 & 833 & 0.827 & 0.335 & 4.9 & 0.054 & 29 \\
\hline $15 / 80$ & 11.6 & 870 & 0.906 & 0.342 & 6.1 & 0.049 & 35 \\
\hline $24 / 80$ & 11.6 & 861 & 0.943 & 0.330 & 5.9 & 0.049 & 40 \\
\hline
\end{tabular}

a Length of the hexagonal unit cell

c Toratal pore volume at $P / P_{0}=0.995$

d Dubinin Micropore volume e Pore diameter BJH method applied to the desorption branch

\section{- Smoking experiment results}

Mixtures were prepared by thoroughly mixing manually the tobacco (3R4F reference tobacco from Kentucky University) with around 4.8 weight \% catalyst. Gas and condensed fractions were analysed. TPM, nicotine, $\mathrm{CO}$ and $\mathrm{CO}_{2}$ results are shown in Tables 2 and 3. Figures 3 and 4 show the reductions obtained in the different families of compounds considered in the gas and TPM fractions.

The effect of removing part of the reaction medium is very small, even certain improvement is observed. This low effect also had been observed in the textural properties developed by the catalysts. According to this, the most of the mother liquor can be eliminated during the hydrothermal treatment obtaining similar results, which is a very important feature for reducing the cost of the equipment and simplifying the process.

On the other hand, increasing the temperature results in a decrease in the reduction ability of nicotine

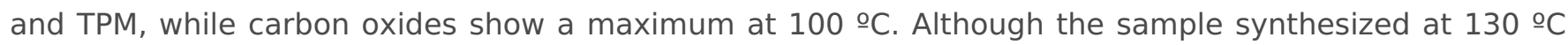
presented slightly lower results in gases and similar in TPM, it is possible to affirm that all samples show similar and very large reduction capacity for most compounds.

For the compounds in gas fraction, no clear trends are observed but all the catalysts obtained are very effective in reducing tobacco smoke compounds. In fact, most families show reductions around $70 \%$ in TPM compounds, carbonyls and phenolic presenting higher values.

A clear growing trend in the reductions is observed, both in the gas and TPM fractions, when increasing the time of hydrothermal treatment. However, the results obtained at $15 \mathrm{~h}$ of hydrothermal treatment are very similar to, or even better than, those obtained at $24 \mathrm{~h} \mathrm{tH}$. These results can be relevant when considering a large-scale production process. 


\begin{tabular}{|c|c|c|c|c|c|}
\hline Sample & TPM (\%) & Gases (\%) & Nicotine (\%) & $\mathrm{CO}_{2}(\%)$ & $\mathrm{CO}(\%)$ \\
\hline $24 / 80^{*}$ & 65.6 & 22.7 & 63.4 & 22.9 & 28.6 \\
\hline $24 / 80$ & 68.1 & 27.8 & 66.8 & 23.4 & 31.0 \\
\hline $24 / 100^{*}$ & 65.0 & 33.1 & 60.6 & 34.8 & 37.3 \\
\hline $24 / 100$ & 67.4 & 44.0 & 61.2 & 38.2 & 39.1 \\
\hline $24 / 130$ & 65.6 & 29.9 & 60.3 & 25.6 & 30.0 \\
\hline
\end{tabular}

Figure 3. Effect of temperature of the hydrothermal treatment and reduction of supernatant liquor on the reduction of families of compounds in tobacco smoke: \begin{tabular}{l} 
a) gas fraction, b) TPM fraction. \\
\hline
\end{tabular}
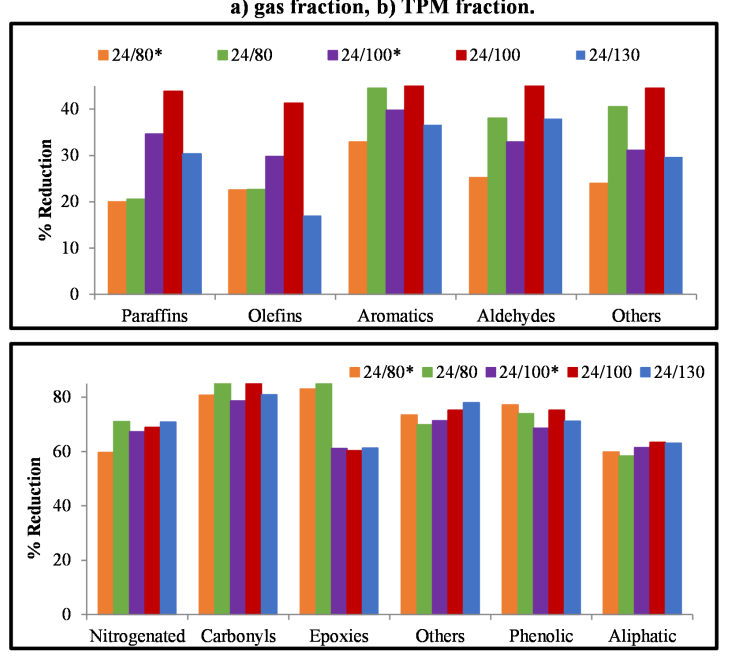

Table 3. Effect of time of the hydrothermal treatment on tar nicotine, $\mathrm{CO}$ and $\mathrm{CO}_{2}$ in tobacco smoke.

\begin{tabular}{cccccc}
\hline Sample & TPM (\%) & Gases (\%) & Nicotine (\%) & $\mathrm{CO}_{2}(\%)$ & $\mathrm{CO}(\%)$ \\
\hline $0 / 80$ & 36.0 & 10.4 & 28.1 & -0.3 & 2.5 \\
$2 / 80$ & 41.9 & 13.8 & 33.9 & 14.1 & 21.0 \\
$6 / 80$ & 60.9 & 17.7 & 55.0 & 18.1 & 24.3 \\
$15 / 80$ & 74.9 & 23.3 & 66.4 & 29.0 & 34.0 \\
$24 / 80$ & 68.1 & 27.8 & 66.8 & 23.4 & 31.0 \\
\hline
\end{tabular}

Figure 4. Effect of time of the hydrothermal on the reduction of families of compounds in tobacco smoke: a) gas fraction, b) TPM fraction.
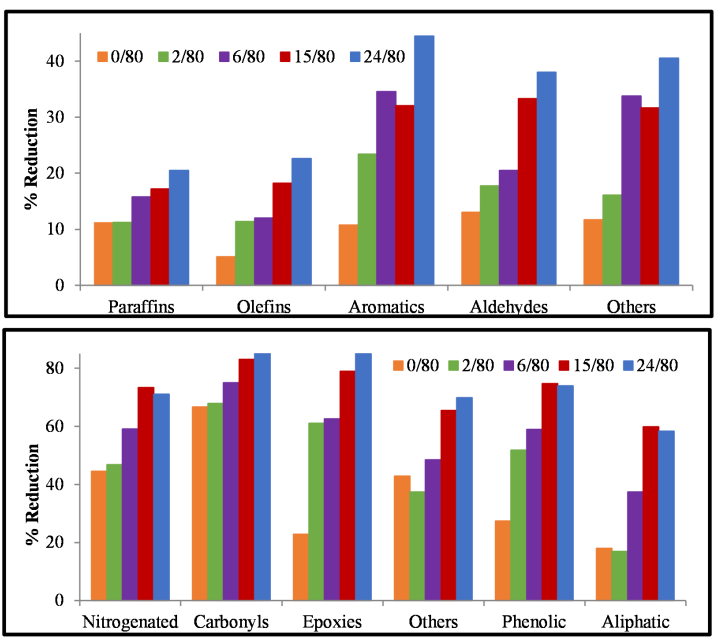

\section{Conclusions}

SBA-15 synthesized in this study show good behaviour as catalysts for reducing the toxicity of tobacco smoke. Furthermore, reducing the amount of mother liquor during the hydrothermal treatment leads to very good results. This would allow the use of smaller volume equipment which is especially relevant when considering that such equipment must stand the pressure generated in the process. On the other hand, good results have also been obtained for the proposed application at all temperatures studied, therefore, the lowest of these $(80 \stackrel{\circ}{\circ})$ could be chosen for large-scale manufacturing. Both issues could have important implications for reducing the manufacturing costs of these materials in large-scale processes. It has been observed that, the apparent density of these powder materials is a very relevant property in the application we are studying since it may be related, in addition to the morphology of the particles, to the way the catalyst particles contact the tobacco strands.

Finally, one aspect that we would like to highlight is that the use of these materials may help in matching the recent suggestion of the FDA to reduce nicotine levels in cigarettes to $0.5 \mathrm{mg} / \mathrm{g}$ of tobacco filler, in order to reach the objective of minimizing levels of addiction.

\section{Acknowledgements}

Authors wish to thank the Spanish Government, project TQ2015-70726-P (MINECO/FEDER) and the Valencian Conselleria d'Educació, Investigació, Cultura I Esport, project PROMETEO/2016/056, and IDIFEDER 2018/009 for financial support.

\section{References}

1. Anh Ngo; Kai-Wen Cheng; Ce Shang; Jidong Huang; Frank J. Chaloupka; Global Evidence on the Association between Cigarette Graphic Warning Labels and Cigarette Smoking Prevalence and Consumption. International Journal of Environmental Research and Public Health 2018, 15, 421, 10.3390/ijerph15030421.

2. Viveka Alfredsson; Håkan Wennerström; The Dynamic Association Processes Leading from a Silica Precursor to a Mesoporous SBA-15 Material. Accounts of Chemical Research 2015, 48, 1891-1900, 10.1021/acs.accounts.5b00165.

3. Pallavi Bhange; Deu S. Bhange; Sivaram Pradhan; Veda Ramaswamy; Direct synthesis of well-ordered mesoporous Al-SBA-15 and its correlation with the catalytic activity. Applied Catalysis A: General 2011, 400, 176-184,

10.1016/j.apcata.2011.04.031. 
4. Haibo Long; Wei Wang; Weiling Yang; Yiran Wang; Hongqiang Ru; Facile and controllable preparation of different SBA-15 platelets and their regulated drug release behaviours. Microporous and Mesoporous Materials 2018, 263, 3441, 10.1016/j.micromeso.2017.11.035.

5. Dongyuan Zhao; Qisheng Huo; Jianglin Feng; Bradley F. Chmelka; Galen D. Stucky; Nonionic Triblock and Star Diblock Copolymer and Oligomeric Surfactant Syntheses of Highly Ordered, Hydrothermally Stable, Mesoporous Silica Structures. Journal of the American Chemical Society 1998, 120, 6024-6036, 10.1021/ja974025i.

6. Pasquale Fulvio; Stanislaw Pikus; Mietek Jaroniec; Tailoring properties of SBA-15 materials by controlling conditions of hydrothermal synthesis. J. Mater. Chem. 2005, 15, 5049, 10.1039/b511346f.

7. Rémy Guillet-Nicolas; François Bérubé; Matthias Thommes; Michael Janicke; Freddy Kleitz; Selectively Tuned Pore Condensation and Hysteresis Behavior in Mesoporous SBA-15 Silica: Correlating Material Synthesis to Advanced Gas Adsorption Analysis. The Journal of Physical Chemistry C 2017, 121, 24505-24526, 10.1021/acs.jpcc.7b06745.

8. Nancy Brodie-Linder; Gilberte Dosseh; Christiane Alba-Simonesco; Fabrice Audonnet; Marianne Impéror-Clerc; SBA15 synthesis: Are there lasting effects of temperature change within the first $10 \mathrm{~min}$ of TEOS polymerization?. Materials Chemistry and Physics 2008, 108, 73-81, 10.1016/j.matchemphys.2007.09.007.

9. Sharon Ruthstein; Veronica Frydman; Shifra Kababya; Miron Landau; Daniella Goldfarb; Study of the Formation of the Mesoporous Material SBA-15 by EPR Spectroscopy. The Journal of Physical Chemistry B 2003, 107, 1739-1748, 10.1021/jp021964a.

10. Anne Galarneau; Hélène Cambon; Francesco Di Renzo; François Fajula; True Microporosity and Surface Area of Mesoporous SBA-15 Silicas as a Function of Synthesis Temperature. Langmuir 2001, 17, 8328-8335, 10.1021/la0105477.

11. Michal Kruk; Mietek Jaroniec; Chang Hyun Ko; Ryong Ryoo; Characterization of the Porous Structure of SBA-15. Chemistry of Materials 2000, 12, 1961-1968, 10.1021/cm000164e.

12. Sara Tasfy; Noor Asmawati Mohd Zabidi; Duvvuri Subbarao; Maizatul Shaharun; Effects of Synthesis Conditions on the Textural and Morphological Properties of Mesoporous Silica (SBA-15). Applied Mechanics and Materials 2013, 446, 201-205, 10.4028/www.scientific.net/amm.446-447.201.

\section{Keywords}

SBA-15; tobacco additive; tobacco toxicity reduction; hydrothermal treatment

(C) 2020 by the author(s). Distribute under a Creative Commans CC BY license 